\title{
Dinámica Directa de Robots Paralelos Utilizando las Ecuaciones de Gibbs-Appell
}

Miguel A. Díaz, Sebastián E. Provenzano, Mary J. Vergara y Rubén D. Chacón Universidad de Los Andes, Escuela de Ingeniería Mecánica, Grupo de Diseño y Modelado de Máquinas, Núcleo la Hechicera, Mérida 5101-Venezuela (e-mail: dmiguel@ula.ve,prse@ula.ve,vmary@ula.ve,rdchacon@ula.ve)

\section{Resumen}

En este trabajo se propone una formulación sistemática que permite resolver el problema dinámico directo de robot paralelos, constituidos por eslabones rígidos y juntas ideales, mediante el uso de las ecuaciones de Gibbs-Appell explícitas, propuestas por Udwadia y Kabala. La metodología permite transformar la resolución del problema dinámico de un sistema mecánico restringido, a la resolución de un sistema conformado por cadenas abiertas, lo cual permite hacer uso de algoritmos eficientes basados en las ecuaciones de Gibbs-Appell. La validación de la metodología se realiza a través de dos ejemplos numéricos.

Palabras claves: sistemas multicuerpo, robot paralelos, ecuaciones Gibbs-Appell, dinámica directa

\section{Forward Dynamic Problem of Parallel Robot by Using Gibbs-Appell Equations}

\begin{abstract}
In this work the use of efficient open chain algorithms based on Gibbs-Appell equations is extended for solving the forward dynamics problem of parallel robots. This extension is carried out by developing a methodology based on the Explicit Gibbs-Appell dynamic equation of motion. The methodology allows transforming the solution of the dynamic problem corresponding to a restricted mechanical system to the solution of a system formed by open chains that permit using the efficient algorithms based on the Gibbs-Appell equations. The methodology was validated using two numerical examples.
\end{abstract}

Keywords: multibody systems, parallel robots, Gibbs-Appell equations, forward dynamic 


\section{INTRODUCCION}

La dinámica directa de sistemas multicuerpo abarca el estudio de las fuerzas aplicadas sistemas mecánicos y su respuesta cinemática en el tiempo. En los últimos años, uno de los sistemas multicuerpo que ha recibido mayor atención por parte de los investigadores en el área, son los robots paralelos (RP). Los RP consisten de una plataforma móvil unida a través de varias cadenas cinemáticas a una plataforma fija. Los RP son muy utilizados en la actualidad sobre todo en ciertas aplicaciones industriales, donde es requerido interactuar con grandes cargas. Este tipo de sistemas presenta ventajas importantes respecto a otros sistemas multicuerpo, por su mayor rigidez y relación de tamaño de sus componentes, lo cual le permite una mayor relación tamaño-carga de trabajo. Estudios de la relación trabajo-carga y ventajas comparativas en las distintas aplicaciones industriales pueden consultarse en Merlet (2000). La principal desventaja de los RP radica en que su configuración en lazo cerrado restringe el movimiento del sistema, lo cual dificulta la obtención sistemática de las ecuaciones que definen su comportamiento dinámico. Los trabajos sobre RP se han enfocado principalmente a la resolución del problema cinemático y relativamente pocos trabajos se enfocan en la dinámica de RP (Tsai, 2000).

Al igual que en los sistemas de cadenas abiertas, diversos principios de la dinámica han sido empleados por los investigadores para hallar las ecuaciones que modelan el comportamiento dinámico de los RP. Es así, por ejemplo, como Do y Yang (1988) y Dasgupta y Mruthyunjaya (1998), utilizaron las ecuaciones de Newton-Euler; Lebrel et al. (1993) y Miller y Clavel (1992), las de Lagrange-Euler; y Wang y Gosselin (1998), Tsai (2000) y Geike y Mcphee (2003) el Principio de los Trabajos Virtuales. Las ecuaciones obtenidas empleando los principios de la dinámica antes mencionados, conllevan a la resolución principalmente de dos tipos de modelos. El primero de ellos se trata de un sistema aumentado, en el cual las ecuaciones de restricción, debido a la configuración cerrada del RP, son incluidas a través de los Multiplicadores de Lagrange (Haug, 1989). En este caso el modelo queda definido por un sistema algebraico diferencial (DAE), donde el número de ecuaciones viene dado por el número de coordenadas generalizadas utilizadas y el número de ecuaciones de restricción. En la obtención de dicho sistema de ecuaciones, que conforma el modelo del sistema, pueden ser empleados algoritmos recursivos eficientes, desarrollados originalmente para cadenas abiertas. Sin embargo, es bien sabido que la solución de un sistema DAE es laboriosa, desde el punto de vista numérico, presentándose la posibilidad de incumplir con las restricciones cinemáticas. El segundo tipo de modelo matemático que se podría conformar, consiste en un sistema reducido, donde las ecuaciones de movimiento están constituidas por un número de ecuaciones ordinarias, igual a los grados de libertad del sistema (GDL). Aunque este procedimiento presenta la ventaja de obtener un número mínimo de ecuaciones y que adicionalmente las ecuaciones obtenidas son ecuaciones diferenciales ordinarias, ésta metodología dificulta la utilización de algoritmos recursivos que permitan determinar sistemáticamente el modelo. Adicionalmente, obliga a la construcción de un modelo diferente para cada RP, haciendo aún más complicada su obtención.

Udwadia y Kabala (1996), partiendo de la forma clásica de las ecuaciones de Gibbs-Appell (G-A) y el principio de mínima acción, proponen las ecuaciones de Gibbs-Appell Explícitas (GAE), las cuales presentan como ventaja principal, que el modelo dinámico está conformado por ecuaciones diferenciales ordinarias. Además permiten que su obtención pueda ser realizada de forma sistemática, empleando inclusive algoritmos recursivos. Una revisión de la literatura del área muestra que las GAE no han sido utilizadas en la resolución del problema dinámico directo (PDD) de RP, por tal motivo, en el presente trabajo se plantea dicho enfoque.

En la siguiente sección se describen las ecuaciones GAE y luego el algoritmo propuesto. Por ultimo, se desarrollan dos ejemplos numéricos y se presentan las conclusiones.

\section{ECUACIONES GIBBS-APPELL EXPLÍCITAS}

El fundamento de las ecuaciones de GAE, se sustenta en la transformación de un sistema restringido de $n+m$ ecuaciones diferenciales ( $n$ es el número de coordenadas generalizadas y $m$ el de ecuaciones de restricción) en un sistema de $n$ ecuaciones diferenciales ordinarias. El sistema de $n+m$ ecuaciones se obtiene al separar el sistema mecánico restringido para obtener un conjunto de varias ca- 
denas abiertas. De esta forma se obtienen un número de $n$ ecuaciones que definen el modelo dinámico de las cadenas abiertas.

$\mathbf{D} \cdot \ddot{q}=\vec{\gamma}$

siendo,

$\mathbf{D}=\left[\begin{array}{ll}\mathbf{D}_{e e} & \mathbf{D}_{e i} \\ \mathbf{D}_{i e} & \mathbf{D}_{i i}\end{array}\right], \ddot{\vec{q}}=\left[\begin{array}{c}\ddot{\vec{q}}_{e} \\ \ddot{\vec{q}}_{i}\end{array}\right]$ y $\quad \vec{\gamma}=\left[\begin{array}{c}\vec{\gamma}_{\mathrm{e}} \\ \vec{\gamma}_{\mathrm{i}}\end{array}\right]$

Donde $\mathbf{D}$ es la matriz de masas y se agrupa en varias submatrices: una asociada a las coordenadas independientes $\mathbf{D}_{i i}$, una correspondiente a las coordenadas dependientes $\mathbf{D}_{e e}$ y una asociada a las coordenadas dependientes e independientes $\mathbf{D}_{i e}$. El vector $\vec{q}$ denota las coordenadas generalizadas y $\vec{\gamma}$ es el vector que contiene los términos de las fuerzas centrifugas, Coriolis, gravedad y externas $(\vec{\tau})$.Al sistema de ecuaciones presentado en la expresión (1) es necesario adicionar las $m$ ecuaciones de restricción:

$\left[\begin{array}{ll}\Phi_{e} & \Phi_{i}\end{array}\right] \ddot{\vec{q}}=\vec{b}$

Siendo $\vec{b}$ el vector que contiene términos dependientes de las velocidades generalizadas, y $\Phi$ el jacobiano de restricciones cinemáticas. Aplicando el principio de mínima acción de Gauss al sistema constituido por las ecuaciones (1) y (3), se obtiene las ecuaciones GAE:

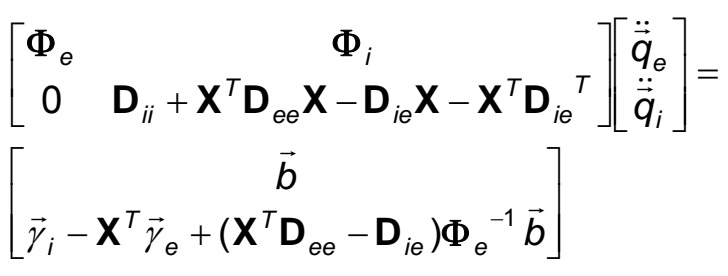

donde $\mathbf{X}=\Phi_{e}^{-1} \Phi_{i}$

Una de las principales razones del uso no extendido de las ecuaciones GAE radica en la dificultad para la obtención, de manera sistemática, de las submatrices independiente y dependiente de la matriz de masas. En este sentido, en la siguiente sección se propone una metodología para la resolución de la dinámica de RP.

\section{RESOLUCIÓN DEL PROBLEMA DINAMICO}

El primer paso en la resolución de la dinámica directa de un RP consiste en separar, las ecuaciones que modelan al robot, en los pares cinemáticos que unen la plataforma móvil. De esta forma se obtiene un sistema conformado por tantas cadenas abiertas como piernas presente el robot. La dinámica de cada pierna se determina mediante la siguiente expresión.

$\left[\mathbf{D}^{k}\right]_{n \times n}\left[\ddot{q}^{k}\right]_{n \times 1}=\vec{\gamma}^{k}{ }_{n \times 1}$

El subíndice $k$ identifica el número de cadenas abiertas y $n$ el número de barras asociadas a la "pierna". Si el RP consta de $k$ piernas, la dinámica directa de las ramas se obtiene por separado de la forma:

$\left.\left[\mathbf{D}^{1}\right]_{n \times n} \mid \ddot{\vec{q}}^{1}\right]_{n \times 1}=\vec{\gamma}^{1}{ }_{n \times 1}$ 


$$
\begin{aligned}
& \left.\left[\mathbf{D}^{2}\right]_{m \times m} \mid \ddot{\vec{q}}^{2}\right\rfloor_{m \times 1}=\vec{\gamma}^{2}{ }_{m \times 1} \\
& \left.\left[\mathbf{D}^{k}\right]_{s \times s} \mid \ddot{\vec{q}}^{k}\right]_{s \times 1}=\vec{\gamma}_{s \times 1}^{k}
\end{aligned}
$$

Las matrices de masas D pueden ser halladas utilizando algoritmos desarrollados para resolver el PDD. En este trabajo se emplean algoritmos basados en las ecuaciones de G-A (Mata et al. 2002a). La separación de la matriz de masas en las componentes dependientes e independientes del RP puede ser realizada de diferentes maneras. En este trabajo se realiza dicha separación seleccionando una coordenada independiente por cada pierna, tal y como se ilustra en las siguientes expresiones:

$$
\begin{aligned}
& \mathbf{D}^{k}{ }_{i i}=\mathbf{D}^{k}{ }_{q i, q i} \\
& \mathbf{D}^{k}{ }_{i e}=\left[\begin{array}{ll}
\mathbf{D}^{k}{ }_{q i, 1 . . q i-1} & \mathbf{D}^{k}{ }_{q i, q i+1 . . n}
\end{array}\right] \\
& \mathbf{D}^{k}{ }_{e i}=\left[\begin{array}{ll}
\mathbf{D}^{k}{ }_{1 . . q i-1, q i} & \mathbf{D}^{k}{ }_{q i+1 . . n, q i}
\end{array}\right] \\
& \mathbf{D}^{k}{ }_{e e}=\left[\begin{array}{ll}
\mathbf{D}^{k}{ }_{1 . . q i-1,1 . . q i-1} & \mathbf{D}^{k}{ }_{1 \ldots q i-1, q i+1 . . n} \\
\mathbf{D}^{k}{ }_{q i+1 . . .1, \ldots q i-1} & \mathbf{D}^{k}{ }_{q i+1 . . n, q i+1 . . n}
\end{array}\right]
\end{aligned}
$$

Luego de obtener la matriz de masas de cada pierna y separarlas según el procedimiento expuesto, la matriz de masas global, ecuación (1), se define:

$$
\begin{aligned}
& \mathbf{D}_{i i}=\left[\begin{array}{ccc}
\mathbf{D}_{i i}^{1} & 0 & 0 \\
0 & \ddots & 0 \\
0 & 0 & \mathbf{D}_{i i}^{k}
\end{array}\right], \mathbf{D}_{i e}=\left[\begin{array}{ccc}
\mathbf{D}_{i e}^{1} & 0 & 0 \\
0 & \ddots & 0 \\
0 & 0 & \mathbf{D}_{i e}^{k}
\end{array}\right] \\
& \mathbf{D}_{e i}=\left[\begin{array}{ccc}
\mathbf{D}_{e i}^{1} & 0 & 0 \\
0 & \ddots & 0 \\
0 & 0 & \mathbf{D}_{e i}^{k}
\end{array}\right], \mathbf{D}_{e e}=\left[\begin{array}{ccc}
\mathbf{D}_{e e}^{1} & 0 & 0 \\
0 & \ddots & 0 \\
0 & 0 & \mathbf{D}_{e e}^{k}
\end{array}\right]
\end{aligned}
$$

Los vectores $\vec{\gamma}$ se determinan de manera eficiente adecuando algoritmos que resuelven el problema dinámico inverso (PDI), tal y como fue propuesto por Walker y Orin (1982). Este procedimiento consiste en anular, en el algoritmo PDI, los términos relacionados con las aceleraciones en los nudos. En este trabajo se emplea el algoritmo desarrollado por Mata et al. (2002b) para resolver el PDI modificado. Los vectores $\vec{\gamma}_{e}, \vec{\gamma}_{i}$, se obtienen de forma simple al seleccionar directamente las filas asociadas a las coordenadas independientes. Para completar el modelo dinámico de RP, es necesario incluir la relación entre las coordenadas dependientes. Esto se lleva se lleva a cabo derivando dos veces respecto al tiempo las ecuaciones cinemáticas de restricción.

$$
\begin{aligned}
& \Phi(\mathbf{q}, t)=0 \\
& \Phi_{q} \dot{\vec{q}}=-\Phi_{t} \\
& \left.\Phi_{q} \ddot{\vec{q}}=-\Phi_{t t}-\left(\Phi_{q} \dot{\vec{q}}\right)_{q} \dot{\vec{q}}-2 \Phi_{q t} \dot{\vec{q}}\right)
\end{aligned}
$$

Los subíndices $t$ y $q$, indican diferenciación respecto al tiempo y a las coordenadas generalizadas respectivamente. Las ecuaciones de restricción en el caso del RP modelan las restricciones en los pares cinemáticos seccionados. El sistema de ecuaciones (7), se determina de forma explícita siguiendo los procedimientos expuestos en Haug (1989). El jacobiano de restricciones $\Phi_{q}$ se obtiene utilizando herramientas de álgebra computacional del programa Maple. El jacobiano es separado indicando las columnas correspondientes a las coordenadas dependientes e independientes, tal y co- 
mo se observa en la ecuación (10). Para su solución numérica las ecuaciones anteriores se codifican en el programa Matlab.

$$
\begin{aligned}
& \Phi_{i}=\left[\begin{array}{lll}
\Phi_{q i}{ }^{1} & \ldots & \Phi_{q i}{ }^{k}
\end{array}\right] \\
& \Phi_{e}=\left[\begin{array}{llll}
\Phi_{1 . . q i-1, q i+1 . . n} & \ldots & \Phi_{1 . . q i-1, q i+1 . . n}
\end{array}\right]^{\top}
\end{aligned}
$$

\section{EJEMPLOS NUMÉRICOS}

Ejemplo Numérico 1. En este ejemplo, Fig. 1, se analiza el desempeño dinámico del RP de 1 GDL sometido a un par variable actuando sobre la barra 1.

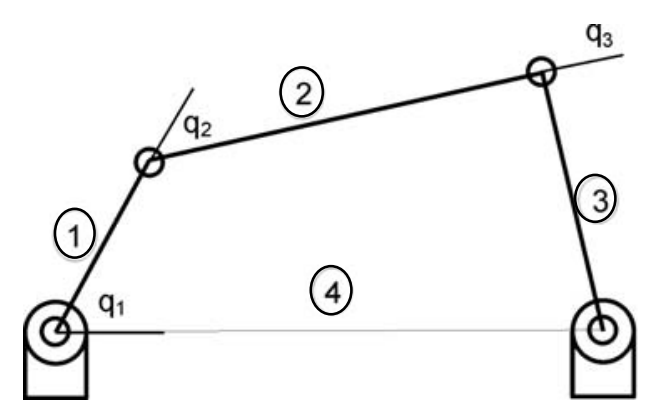

Fig. 1: Robot paralelo de $1 \mathrm{GDL}$

Los datos que definen el par variable a través del tiempo, se determinó desarrollando una rutina que resuelve el PDI utilizando el Principio de los Trabajos Virtuales. Se obtienen datos del par aplicado a la barra 1 cuando el RP es conducido cinemáticamente por una velocidad angular constante de 1 $\mathrm{rad} / \mathrm{seg}$. El tiempo de simulación es de 2.5 segundos. La configuración inicial del RP es: $\theta_{1}=0.785$ $\mathrm{rad}, \theta_{2}=0.323 \mathrm{rad}$ y $\theta_{3}=-1.892 \mathrm{rad} . \omega_{1}=1 \mathrm{rad} / \mathrm{s}$. Las dimensiones de las barras: $L_{1}=1 \mathrm{~m}, L_{2}=3 \mathrm{~m}$, $L_{3}=7 / 4 \mathrm{~m}$ y $L_{4}=3 \mathrm{~m}$, siendo $L_{4}$ la longitud de la barra fija al marco de referencia. Las masas: $m_{1}=1 \mathrm{~kg}$, $\mathrm{m}_{2}=2 \mathrm{~kg}, \mathrm{~m}_{3}=1.5 \mathrm{~kg}$ y los momentos respecto al centro de gravedad del cuerpo, $\mathrm{I}_{1}=1 \mathrm{kgm}^{2}, \mathrm{I}_{2}=2 \mathrm{kgm}^{2}$ $\mathrm{y}_{3}=1.5 \mathrm{kgm}^{2}$.

A partir de los datos iniciales y las propiedades de masa, la ecuación (4) es resuelta mediante un código implementado en el ambiente Matlab/Maple. El código determina el valor de las aceleraciones y éstas se integran para obtener las velocidades y posiciones angulares independientes. Las velocidades y posiciones dependientes se obtienen luego a partir de las velocidades y posiciones independientes. El sistema de ecuaciones diferenciales ordinarias es resuelto mediante la función de Matlab ode113, la cual se basa en el algoritmo de Adams-Bashforth-Moulton. El algoritmo es de tipo multipaso adaptativo. Para determinar la tolerancia adecuada del integrador, se realizaron simulaciones para distintos valores, encontrando que a partir de tolerancias de integración de $10^{-6}$ se obtuvieron resultados similares. En la Fig. 2 se muestra la variación del ángulo de la barra 1 respecto al tiempo.

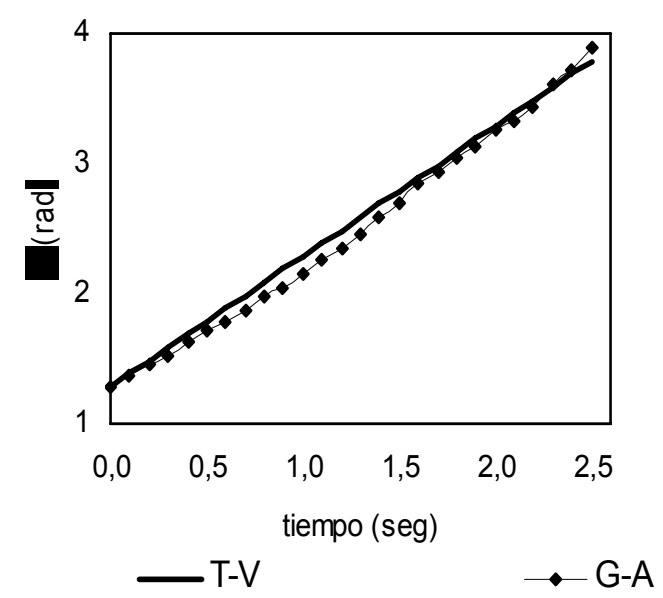


Fig. 2: Posición Angular Eslabón 1

Se aprecia en la Fig. 2 que el comportamiento del ángulo es lineal y ascendente, en concordancia con la condición cinemática bajo la cual fue obtenida el par aplicado (velocidad angular constante). Se evalúa igualmente la diferencia entre las configuraciones de entrada al PDI y los resultados obtenidos del procedimiento propuesto, los cuales son similares durante el intervalo de integración.

Ejemplo Numérico 2. En este ejemplo se estudió el comportamiento dinámico de una RP de 3 GDL (Khan et al., 2005). El sistema está constituido por una base móvil unida por medio de tres cadenas abiertas al marco de referencia. Los actuadores (motores) del sistema se encuentran ubicados en el primer eslabón de cada cadena. El vector gravedad es perpendicular al plano de movimiento de la RP. La separación del RP conlleva a la obtención de un total de 3 cadenas abiertas en las cuales una de las cadenas esta formada, adicionalmente a los dos eslabones, por el eslabón asociado a la coordenada $q_{3}$ mostrada en la Fig. 3 . En este ejemplo se evalúa el comportamiento dinámico del RP bajo la acción de pares constantes, siendo sus valores: ${ }_{1}=0.1 \mathrm{Nm}, \quad{ }_{4}=0.1 \mathrm{Nm}$ y ${ }_{6}=-0.1 \mathrm{Nm}$. Las posiciones iniciales son: $\theta_{1}=1.047 \mathrm{rad}, \theta_{2}=-0.865 \mathrm{rad}, \theta_{3}=1.116 \mathrm{rad}, \theta_{4}=2.751 \mathrm{rad}, \theta_{5}=-1.424 \mathrm{rad}, \theta_{6}=$ $0.679 \mathrm{rad}$ y $\theta_{7}=-2.718 \mathrm{rad}$. La velocidad inicial del sistema es nula. Las dimensiones de las barras

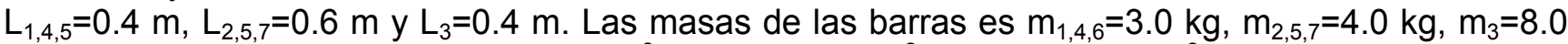
$\mathrm{kg}$ y momentos de Inercia $\mathrm{I}_{1,4,6}=0.04 \mathrm{~kg} \mathrm{~m}^{2}, \mathrm{I}_{2,5,7}=0.12 \mathrm{~kg} \mathrm{~m}^{2}$ y $\mathrm{I}_{3}=0.0817 \mathrm{~kg} \mathrm{~m}^{2}$.

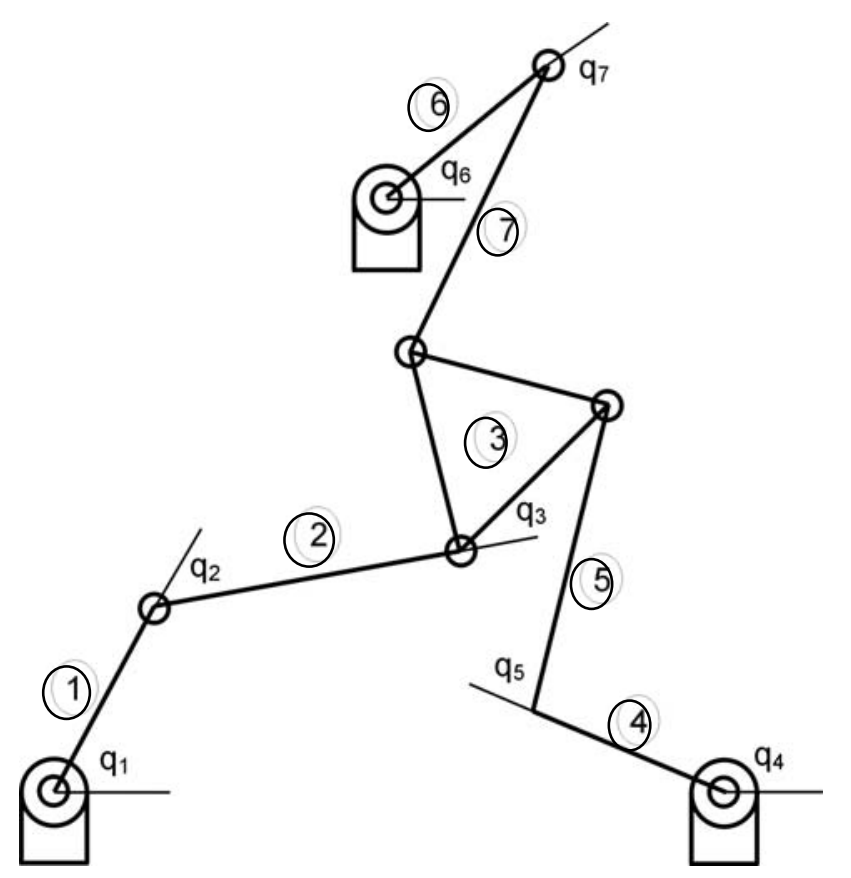

Fig. 3: Robot paralelo de $3 \mathrm{GDL}$

La Fig. 4 muestra la comparación resultantes de simular el sistema mecánico en el programa Working Model 2D (WM). Se aprecia en las figuras (4) y (5) que los resultados arrojados son similares durante el intervalo de simulación.

Con el objeto de evaluar el tiempo de ejecución del algoritmo propuesto, se resuelve el PDD utilizando las ecuaciones de G-A e incluyendo las ecuaciones de restricción mediante los multiplicadores de Lagrange (Tabla 1). El sistema DAE fue resulto mediante la rutina ode23t de Matlab, recomendado para este tipo de sistemas. Los resultados obtenidos de ambos procedimientos fueron idénticos para el intervalo de simulación, aunque se puede observar que para el procedimiento basado en las GAE, el tiempo de cómputo es menor.

Tabla 1: Evaluación del tiempo de cómputo

\begin{tabular}{lcc}
\cline { 2 - 3 } & $\begin{array}{c}\text { \# de evaluac. fun- } \\
\text { ción de integración }\end{array}$ & $\begin{array}{c}\text { tiempo } \\
\text { (seg) }\end{array}$ \\
\hline G-A aumentado & 61 & 3.4219
\end{tabular}




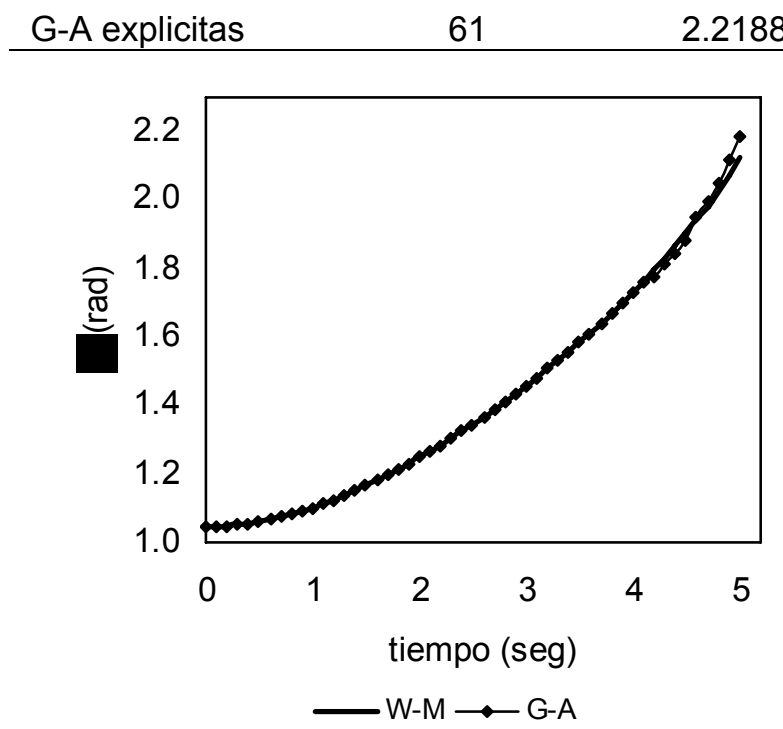

Fig. 4: Resultados GAE vs WM

\section{CONCLUSIONES}

En este trabajo las ecuaciones GAE, planteadas por Udwadia y Kabala (1996), fueron empleadas para la resolución del problema dinámico directo de RP. Se propuso un procedimiento sistemático que permite obtener las diferentes matrices y vectores que las componen de una manera sistemática empleando procedimientos eficientes que resuelven los problemas dinámicos de cadenas abiertas. Los resultados del algoritmo fueron verificados mediante la resolución de dos ejemplos numéricos. Mediante un ejemplo de comparación entre las GAE y el procedimiento basado en multiplicadores de Lagrange se demostró que el primero arroja resultados precisos en menor tiempo.

\section{AGRADECIMIENTOS}

Los autores de este trabajo agradecen al CDCHT por la colaboración y financiamiento otorgados a este trabajo designado bajo el código: I-801-04-02-A

\section{REFERENCIAS}

Dasgupta B. y T.S. Mruthyunjaya; "Closed Form Dynamic equations of the General e Stewart Platform through the Newton-Euler Approach," Mechanisms and Machine Theory, 33(8), 993-1012 (1998).

Do W. Q. y D.C Yang; “Inverse Dynamic Analysis and Simulation of a Platform Type of Robot”, Journal of Robotic Systems," 53(5), 209-227 (1988).

Geike, T. y J. McPhee; "Inverse dynamic Analysis of Parallel Manipulators with Full mobility", Mechanism and Machine Theory, 38, 549-562 (2003).

Haug E., "Computer Aided Kinematics and Dynamics of Mechanical System", Allyn and Bacon, USA (1989).

Khan W. A., V.N. Krovi, S.K. Saha y J. Angeles; "Modular and Recursive Kinematics and Dynamics for Parallel Manipulators". Journal of Multibody System Dynamics, 14, 419-455 (2005).

Lebret G., K. Liu y F.L. Lewis; "Dynamic Analysis and Control of a Stewart Platform Manipulator", Journal Robotics Systems, 10 (5), 629-655 (1993). 
Mata V., S. Provenzano, F. Valero y J.I. Cuadrado; “Serial-Robot Dynamics Algorithms for Moderately Large Numbers of Joints", Mechanism and Machine Theory, 37(8), 739-755 (2002a).

Mata V., S. Provenzano, J.I. Cuadrado y F. Valero; "Inverse Dynamic Problem in Robots using GibbsAppell Equations", Robotica. 20(1), 59-67 (2002b).

Merlet J.M., "Parallel Robots", Kluwer Academia Publishers, London (2000).

Miller K. y R. Clavel; "The Lagrange-based model of Delta-4 robot dynamics". Robotersysteme. Springer-Verlag. 8(4), 49-54 (1992).

Tsai, L.-W.; "Solving the Inverse Dynamics of a Stewart-Gough Manipulator by the Principle of Virtual Work", ASME J. Mechanical Design, 122(1), 3-9 (2000).

Udwadia F.E. y R.E. Kabala; "Analytical Dynamic a New Approach". Cambridge University Press, Cambridge (1996).

Walker W. y D.E. Orin; "Efficient Dynamic Computer Simulation of Robotic Mechanisms". Journal of Dynamic Systems. Meas., Contr., 104, 205-2011 (1982).

Wang, J. y C.M. Gosselin; "A New Approach for the Dynamic Analysis of Parallel Manipulators", Multibody Sysem Dynamics, 2(3), 317-334 (1998). 